\title{
FUZZY IMAGE SEGMENTATION FOR URBAN LAND-COVER CLASSIFICATION
}

\author{
I. Lizarazo $^{\mathrm{a}, *}$, J. Barros ${ }^{\mathrm{b}}$

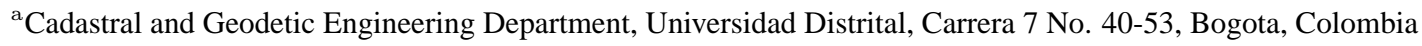 \\ ilizarazo@udistrital.edu.co \\ ${ }^{\mathrm{b}}$ School of Geography, Birkbeck College, University of London, Malet Street, London, WC1E 7HX UK \\ j.barros@bbk.ac.uk
}

KEY WORDS: Segmentation, classification, land-cover, image-regions, image-objects, fuzzy

\begin{abstract}
:
In this paper a general fuzzy approach for segmentation-based classification is proposed. Traditional segmentation techniques focus on partitioning imagery into image-objects with well-defined boundaries. Instead, the proposed methodology aims to produce and analyze fuzzy image-regions expressing degrees of membership to different target classes. This approach, called Fuzzy Image-Regions Method (FIRME), is suitable to deal with the spectrally and spatially complexity of urban landscapes. The main stages of the FIRME approach are described, including techniques to produce such regions, alternatives to measure region attributes, and a number of methods for defuzzification. The FIRME method is tested for an urban classification experiment using multi-spectral imagery from Bogota, Colombia. Results suggest that, in complex environments, the FIRME method may be a suitable alternative to hard segmentation as it performs well in discriminating between spectrally mixed geographic objects.
\end{abstract}

\section{INTRODUCTION}

Pixel-wise approaches for image classification are not usually suitable to solve spectral mixture problems which are often found in different remote sensing applications (Mesev, 2003), (Thomas et al., 2003). Recent research clearly shows the advantages of integrating spatial dimension to spectral features by using segmentation based classification methods and, hence, focusing into image-objects instead of pixels (Jensen, 2006), (de Jong and Freek, 2006). However, producing meaningful and well defined imageobjects able to represent the structural properties of the target classes is a time consuming, iterative, and not always well succeed process (Schiewe et al., 2001), (Lang et al., 2006). This paper aims to demonstrate that a more generic fuzzy image segmentation approach is appropriate and desirable when dealing with complex environments like urban landscapes. This paper is organized as follows. Section 2 reviews fuzzy segmentation concepts and introduce the general functional model of our approach. Section 3 demonstrates the potential of the proposed approach with a land-cover classification experiment in an urban environment. Section 4 discusses the main experimental results. Section 5 presents the concludsions.

\section{FUZZY IMAGE SEGMENTATION}

\subsection{Fuzzy Classification: an overview}

Fuzzy classification has been applied in remote sensing for natural phenomena that are distributed gradually and continuously over space. In such cases, there are no hard boundaries dividing geographic objects (Roberts et al., 2001). In other cases, well defined objects appear blurred in images because of sensor noise, shading and highlights. Thereby, a fuzzy classification may be more appropriate than representing reality through sharp objects and crisp classes (Cheng et al., 2001). Fuzzy classification methods assign gradual membership of pixels to classes measured as degrees in $[0,1]$. This gives the flexibility to represent pixels that belong to more than one class.

The concept of these membership degrees is based on the definition and interpretation of fuzzy sets (Zadeh, 1965). An element of a fuzzy set can have different degrees of membership to the set. It can be full member (100\% membership) or a partial member (between $0 \%$ and $100 \%$ membership). That is, the membership value assigned to an element is no longer restricted to two values, but can be 0,1 or any value in-between. The mathematical function that defines the degree of an element's membership in a fuzzy set is called membership function. Since membership to classes is fuzzy, there is no single label indicating to which cluster a pixel belongs. Instead, fuzzy classification methods associate a fuzzy label vector to each pixel $x_{j}$ which states its membership to each one of $c$ classes:

$$
U_{j}=\left(\mu_{1 j}, \ldots, \mu_{c j}\right)^{T}
$$

The $c \times \mathrm{x} n$ matrix $U=\left(\mu_{i j}\right)=\left(\mu_{1}, \ldots, \mu_{n}\right)$ is called a fuzzy partition matrix. Based on this notion it is possible to handle ambiguity of classes assignments when they are overlapping or badly delineated (Kruse et al., 2007).

One of the most commonly used fuzzy classification methods is the unsupervised Fuzzy c-Means (FCM) classifier (Fisher and Arnot, 2007). Fuzzy interpretation of satellite imagery is usually focused on Type-1 fuzzy sets, that is, the membership of pixels to classes is defined by a crisp value in the range $[0,1]$. Recent research uses lower and upper bounds to describe the degrees of pixels belonging to one specific class. By using a vector with minimum, average and maximum membership values, a Type-2 fuzzy set is produced (Mendel, 2001). Fuzzy sets concepts have been useful to define classification schemas for interpretation of environmental phenomena (Fisher and Arnot, 2007). In this paper, we use fuzzy sets to generalize the image segmentation process as it has been suggested in the computer vision community (Bezdek et al., 1999).

\subsection{Fuzzy image-regions}

Fuzzy segmentation is understood here as the process of converting a given multi-spectral image into a fuzzy set by indicating, for each pixel, the degree of its membership to every target class. Thus, fuzzy segmentation produces $n$ images, i.e. a grey-level image for every class, where pixels show the possibility of belonging to one or more of the target classes. Each output image is 


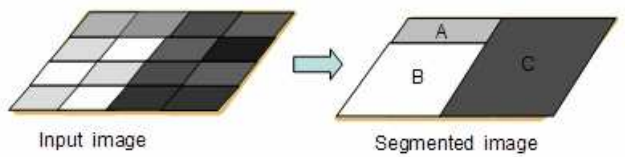

Figure 1: Traditional image segmentation produces imageobjects with well defined boundaries.

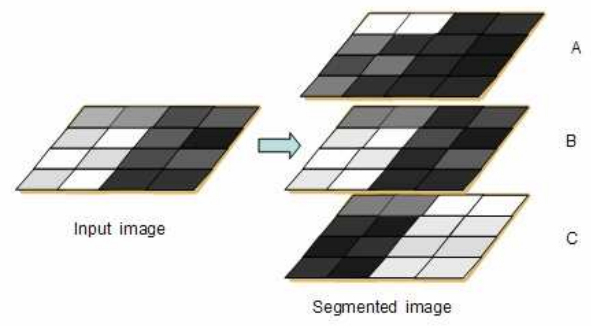

Figure 2: Fuzzy image segmentation produces a set of imageregions whith uncertain boundaries.

a thematic grouping of pixels based on their degrees of similarity to the training samples. However, this is not a discrete grouping, where pixels are allocated to one or another class and contiguous pixels are aggregated to create polygons. This is a new kind of grouping which can be referred to as fuzzy image-regions.

A fuzzy image-region has an uncertain thematic description and also an uncertain spatial extent, i.e. it is a fuzzy-fuzzy imageregion (Cheng et al., 2001). As such, a fuzzy image-region is not defined by a predefined outline as traditional image-objects do. Instead, a fuzzy image-region is a fuzzy set of pixels over a two-dimensional domain. All pixels are assigned membership grades in the range $[0,1]$ indicating the extent to which each pixel belongs to the region. Thus, a membership grade 1 indicates it belongs fully to the region. A membership grade 0 indicates that a pixel does not belong to the region (Verstraete et al., 2007). Figures 1 and 2 illustrate the main difference between hard segmentation which produces one single image at every scale, and fuzzy segmentation which produces a set of as many images as target classes exist. In this paper, fuzzy segmentation is understood as the supervised process which takes early advantage of user knowledge.

A simple way of processing fuzzy-regions is converting them into a single layer, that is, aggregating into a single image in order to produce a complete partition of the area into non-overlapping regions. Although this way does not exploit the information-rich content of the fuzzy regions, it provides a quick method to produce hard classes. Two basic alternatives used in such approach are either: (i) a conditional boundary is set to define explicitly the spatial extent of the fuzzy regions and allocate each pixel to a specific land cover class; or (ii) a clear boundary cannot be defined but there might be transition zones between objects (Cheng et al., 2001). In the first option, the initial set of fuzzy-fuzzy (FF) image-regions may be transformed into crisp-fuzzy (CF) imageobjects. In the second option, the output are converted into fuzzycrisp (FC) objects. On one hand, CF image-objects have crisp boundaries and fuzzy interiors (i.e. memberships must be kept within a certain range in order to avoid overlap between classes). On the other hand, FC image-objects have uncertain boundaries and certain cores (i.e. zones in which membership equals 1) (Cheng et al., 2001). Although this aggregation-based approach has been useful in previous studies, we propose a different way for managing the uncertainty of fuzzy regions. In essence, we

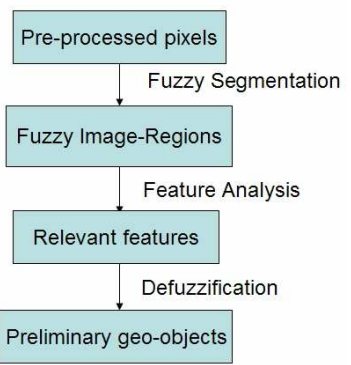

Figure 3: Image classification workflow using the FIRME method.

argue that, following the object-based image analysis approach, fuzzy image-regions properties and relationships should be measured in order to include contextual properties in the subsequent classification stage.

\subsection{FIRMEs functional model}

Figure 3 shows a simplified workflow diagram of our method FIRME, a generic way for implementing image classification using fuzzy image-regions. It depicts a sequential development of three stages: (i) fuzzy segmentation, (ii) feature analysis and (iii) defuzzification. Note that knowledge inputs and feedback loops are not shown in the diagram. The overall process of image classification is based on the concept of supervised pattern recognition, that is, that given a certain number of classes it is necessary to allocate a new individual to one of these classes (Duda et al., 2001). Typically, the classes of a certain number of individuals are known. These individuals, often referred to as a training set, are used for selecting the relevant features or attributes of the individuals and the algorithms for the class recognition. Next sections will discuss the main stages of the FIRME approach for image classification.

2.3.1 Fuzzy segmentation: The objective of this process is to build fuzzy image-regions from the pre-processed (ambiguous) pixels. As explained above, the fuzzy image-regions have values restricted to the range $[0,1]$. Such values represent degrees of belonging of every pixel to the classes under study. In any pattern recognition application, a number of methods for inferring fuzzy memberships from labelled training samples to new data are available (i.e. inference, rank ordering, neural networks, genetic algorithms and inductive reasoning) (Ross, 2004). Many of these methods have been used for remote sensing image classification (Lu and Weng, 2007) and provide a range of capabilities to deal with incomplete or imprecise data. Even more, they may be used to produce fuzzy image-regions. Once a set of membership grey-level images has been produced using any of the existing methods, there will be one fuzzy image-region available for each target class and the subsequent analysis may continue.

2.3.2 Feature Analysis: This process aims to define, select and extract a relevant set of image-regions properties and relationships suitable to infer appropriate decision rules and resolve the spectral ambiguity in land-cover classes. By default, the membership values of regions to target classes are part of the attributes under study. In addition, intersection of fuzzy image-objects may be conducted to detect problematic zones. A useful option for doing this is the confusion index (CI) (Burrough et al., 1997):

$$
C I=1-\left[\mu_{\max i}-\mu_{(\max -1) i}\right] .
$$

where $\mu_{\max i}$ and $\mu_{(\max -1) i}$ are, respectively, the first and second largest membership value of the $i^{t h}$ pixel. The $C I$ measures 
the overlapping of fuzzy classes at any point and provides insight for further investigating the sites with high membership values to more than one class (Bragato, 2004). $C I$ values are in the range $[0,1]$, values closer to 1 describe zones where overlapping is critical.

Other useful indexes to analyze overlapping fuzzy-regions may be adapted from the crisp realm (i.e. indexes commonly used for comparison of raster maps). An index used in the present study is the absolute normalized difference index (ANDI):

$$
A N D I=\left|\mu_{i A}-\mu_{i B}\right|
$$

where $\mu_{i A}$ and $\mu_{i B}$ are the membership values of the $i^{t h}$ pixel to the classes A and B, respectively. The ANDI index measures the overlapping existing between two specific classes.

Although the concept of fuzzy regions or objects has been used in geographic information science for some time, practical implementation of specific methods suited to the spatial realm has only recently started being developed (Verstraete et al., 2007). Well known generic fuzzy operators include: union (UNI), intersection (INT), complement (COM), difference (DIF), and the principles of middle included (MID) and contradiction (CONT), and a number of specific operators for defuzzification, for instance alpha cut (ALP) and intensification (INS) (Ross, 2004). Geometric operations (surface area, distance to a fuzzy region) and specific geographic operations (minimum bounding rectangle, convex hull) are much more complex as a recent implementation has demonstrated (Verstraete et al., 2007).

Also, a number of ways of measuring shape of spatial vague objects have been recently proposed (Chanussot et al., 2005) (Dilo et al., 2006). Modelling and query of spatial vague objects using shapelets have been proposed by (Zinn et al., 2007) and might extend the FIRME method. Although the suitability of using these operators on the fuzzy image-regions studied here still remain to be investigated, their application may enhance the feature vector to be used to inferring appropriate decision rules for discriminating target classes.

2.3.3 Defuzzification: The objective of this process is to in fer and apply decision rules to assign full membership of the fuzzy regions to the target land-cover classes. General defuzzification techniques include: max membership principle, centroid method, weighted average method and mean max membership (Ross, 2004). A simple method of defuzzification - not exploiting feature analysis - uses a logical union operator like the fuzzy t-conorm MAX operator (Ross, 2004):

$$
\mu_{i 1} U \mu_{i 2} \ldots U \mu_{i c}=\max \left(\mu_{i 1}, \mu_{i 2}, \ldots, \mu_{i 3}\right)
$$

where $\max ()$ indicates the largest membership value of the $i^{t h}$ pixel or neighborhood.

As it was suggested before, a more elaborated option, akin to GEOBIA approach, is to enrich the feature vector of memberships with additional attributes of the fuzzy image-regions. Then, a variety of inferential statistical learning methods may be used for accomplish the defuzzification stage (Lizarazo and Elsner, 2008). As it was suggested, users of specific applications have to make decisions about suitable methods to be applied to solving a particular problem. In the next section, one implementation of the FIRME method for an urban land-cover classification experiment is reported.

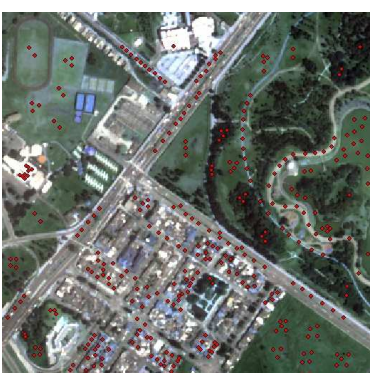

(a)

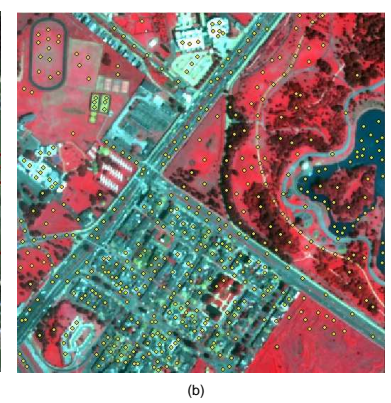

(b)
Figure 4: Colour compositions of QuickBird image data: (a) True Colour (RGB321) ; False Colour (RGB432).

\section{DATA AND METHODS}

\subsection{Data}

The image used is a QuickBird multi-spectral dataset covering a small urban area in Bogota (Colombia). QuickBird imagery is adquired by Digital Globe ${ }^{T M}$ in four bands: blue $(479.5 \mathrm{~nm})$, green $(546.5 \mathrm{~nm})$, red $(654 \mathrm{~nm})$ and near infra-red $(814.5 \mathrm{~nm})$. The input data set is 352 columns x 344 rows. Spatial resolution is $2.44 \mathrm{~m}$ and radiometric resolution is 11 bits. Spectral bands are referred here to as $b 1$ (blue), $b 2$ (green), $b 3$ (red) and $b 4$ (near infrared). Figure 4(a) and (b) show colour compositions in true colour (RGB321) and false colour (RGB432).

\subsection{Methods}

The FIRME implementation in this paper relies on two statistical inferential methods: Generalized Additive Models (GAM) and Support Vector Machines (SVM). GAMs and SVMs are generalizations of linear models (LM) which are used widely in most branches of science (Hastie et al., 2001). Linear models are statistic models in which a univariate response is modelled as the combination of a linear predictor and a zero mean random error term. In the equation 5, a variable response datum, $y i$, is treated as an observation on a random variable, $Y_{i}$, with $E\left(Y_{i}\right)$ as expectation, the $\epsilon_{i}$ as zero mean random variables, and the $\beta_{i}$ are model parameters, the values of which are unknown and will need to be estimated using training data:

$$
E(Y i)=\beta_{0}+x_{i} \beta_{1}+z i \beta_{2}
$$

where $Y i=E(Y i)+\epsilon_{i}$, is a linear model in which $y$ depends on predictor variables $x$ and $z$.

A key feature of a linear model is that the linear predictor depends linearly on the parameters. Statistical inference with such models is usually based on the assumption that the response variable has a normal distribution (Wood, 2006).

Generalized linear models (GAM) allow the expected value of the response to depend on a smooth monotonic function of the linear predictor. Similarly, the assumption that the response is normally distributed is relaxed by allowing it to follow any distribution from the exponential family (i.e. normal, Poisson, binomial, gamma). Moreover, a GAM is a GLM in which part of the linear predictor is specified in terms of a sum of smooth functions of predictor variables. The exact parametric form of these functions is unknown, as is the degree of smoothness appropriate for each of them. Statisticians state that, by going from linear models through GLMs to GAMs, such models become better able to describe the reality and the methods for inference more consistent but less precise (Wood, 2006). 
Table 1: Land-cover classes code and description

\begin{tabular}{ccc}
\hline Code & Land-cover class & Observations \\
\hline 148 & Roads & \\
180 & Rooftops 1 & Medium reflectance \\
183 & Rooftops 2 & High reflectance \\
311 & Grass & \\
325 & Trees & \\
521 & Water Body & \\
731 & Bare Soil &
\end{tabular}

On the other hand, Support Vector Machines (SVM) are robust classifiers which transform the original feature space into a new one using a kernel function, and then use a selected number of samples located in the boundaries of classes- as support vectors to discriminate target classes. Available kernels include: linear, polynomial, radial basis function and sigmoid. The kernel transformation (or kernel trick as many authors refers to it) allows finding a new feature space in which linear hyper-planes are appropriate for class separation. Separating boundaries are nonlinear in the original feature space (Hastie et al., 2001). SVMs are becoming a popular technique for regression and classification tasks.

For the Fuzzy Segmentation stage, a GAM model was fitted using a small training sample whose class labels are known. Training sample, shown in Figure 4(a), comprises 324 pixels which account for less than $0.5 \%$ of the image size. We use an additive approach to model the presence/absence of every land-cover class $c_{i}$, as

$$
\operatorname{logit}(E(Y i))=f\left(b 1_{i}, \ldots, b n_{i}\right)
$$

where $\operatorname{logit}(E(Y i))=\log (E(Y i) /(1-E(Y i))), f$ is a smooth function of the principal component transformation of the multispectral variables, $b 1_{i}, \ldots, b n_{i}$ are $n$ predictor variables, and $c i$ is a binomial function $(1, E(Y i))$.

For the Feature Analysis stage, $\mathrm{R}$ functions were written in order to compute $\mathrm{CI}$ index for the whole set of fuzzy-regions, and the ANDI indexes for the pairs of most problematic classes. Then, fuzzy membership of image-regions plus these indexes were used as feature vector.

For the final Defuzzification stage, a Support Vector Machine was used to discriminate final crisp objects. We use a radial basis function to transform from the original feature space to a new one linear space, to be able to assign a crisp land-cover class $c_{i}$ using the fuzzy predictors, as indicated in this model:

$$
E(Y i)=S V M\left(K\left(\mu_{i 1}, \ldots, \mu_{i c}, \varnothing_{1}, \ldots \emptyset_{n}\right)\right)
$$

where $\mathrm{K}$ is a radial basis kernel applied to $c$ fuzzy image-regions $\left(\mu_{i c}\right)$ and $n$ fuzzy operators $\left(o_{n}\right)$, in order to find SVM vectors able to classify $c_{i}$ as a multinomial function of $(E(Y i))$. As a final step, an evaluation of thematic accuracy was conducted using a testing sample, shown in yellow colour in Figure 4(b), accounting for $0.5 \%$ of the image size. Classs labels follow the USGS Anderson land-cover codes (Anderson et al., 1976) shown in Table 1.

FIRME's implementation was done using $R$, a free software environment for statistical computing and graphics (Bivand et al., 2008). Besides the $R$ base package which provides basic statistical capabilities, additional packages like rgdal, $s p$ and maptools, gam and $e 1071$ were used. They provide, respectively, functions for reading and writing images in standard formats, creating and manipulating spatial classes, building generalized additive models and applying machine learning algorithms.

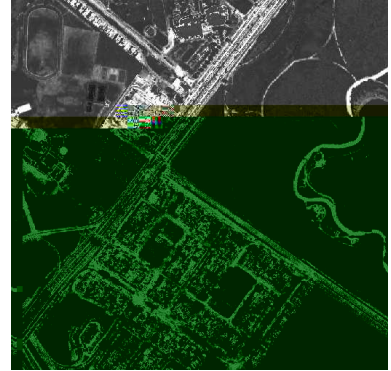

(a)

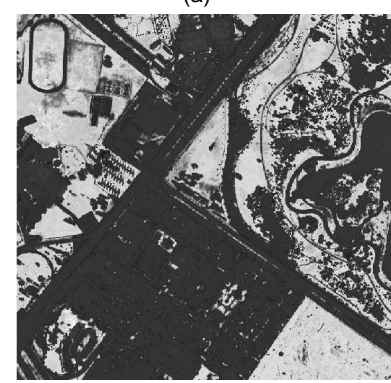

(c)

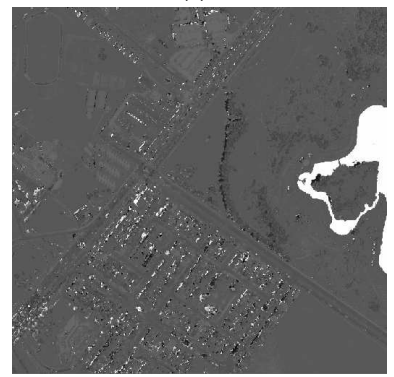

(e) (b)

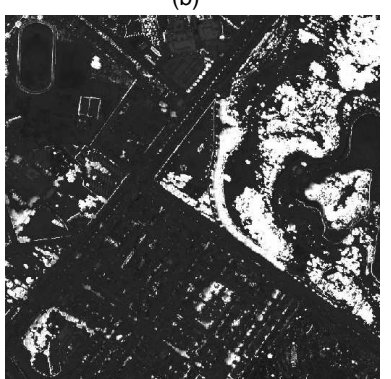

(d)

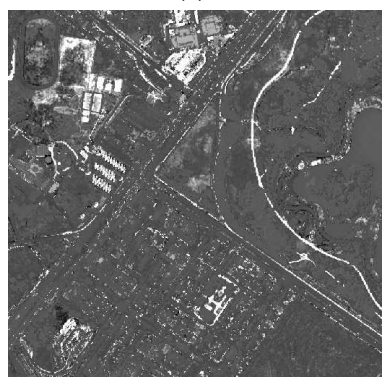

(f)

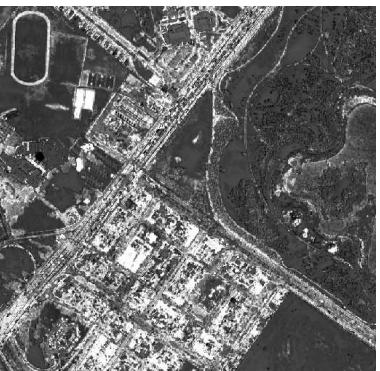

Figure 5: Fuzzy Image-Regions representing membership to land-cover classes. (a) Roads, (b) Rooftops, (c) Grass, (d) Trees, (e) Water, (f) Bare Soil. Light tones represent high degrees of membership.

\section{RESULTS AND DISCUSSION}

In the Fuzzy Segmentation stage, a GAM model was fitted using the following formula:

$$
L A N D \cdot A B C=s(b 1+b 2+b 3+b 4+b 5+b 6)
$$

where $L A N D . A B C$ stands for each one of the different landcover classes (i.e LAND.148, LAND.180, and so on), $s$ is a smooth function, $b 1, b 2, b 3$ and $b 4$ represent the principal components transformation of the original multi-spectral channels, and $b 5$ and $b 6$ represent the $x$ and $y$ coordinates normalized to the range $[0,1]$.

Quality indicators of the GAM model fitting, that is, Null deviance, Residual deviance and Akaike Information Criteria (AIC) of each predictor variable, are shown in Table 2 . Note that model fitting quality for natural classes (grass, trees, water and bare soil) outperforms quality for artificial classes (roads and rooftops).

Fuzzy Image-Regions obtained for every target land-cover class are shown in Figure 5: (a) Roads, (b) Rooftops, (c) Grass, (d) Trees, (e) Water and (f) Bare Soil. Light tone represents high degrees of membership.

In the Feature Analysis stage, standard fuzzy operations were conducted in order to produce the global confusion index $(\mathrm{CI})$ and to evaluate the Absolute Normalized Difference Index (ANDI) 
Table 2: GAM models' quality indicators

\begin{tabular}{ccccc}
\hline Code & Null dev. & Resid. dev. & AIC & \\
\hline 148 & 390.23 & 100.23 & 74 & 184.22 \\
180 & 377.69 & 121.69 & 67 & 201.68 \\
183 & 88.93 & 0 & 100 & 50.00 \\
311 & 305.48 & 0 & 100 & 50.00 \\
325 & 165.26 & 0 & 100 & 50.00 \\
521 & 175.30 & 0 & 100 & 49.99 \\
731 & 132.76 & 0 & 100 & 50.00 \\
\hline
\end{tabular}

Table 3: Confusion matrix for land-cover classification using FIRME method. $\mathrm{PCC}=0.86$. Kappa value $=0.82$

\begin{tabular}{ccccccc}
\hline Map & Road & Roof & Grass & Trees & Water & Soil \\
\hline Road & 135 & 30 & 1 & 5 & 0 & 1 \\
Roof & 18 & 116 & 0 & 1 & 0 & 7 \\
Grass & 1 & 1 & 60 & 0 & 0 & 0 \\
Trees & 0 & 0 & 1 & 43 & 0 & 0 \\
Water & 0 & 0 & 0 & 0 & 34 & 0 \\
Soil & 0 & 3 & 0 & 0 & 0 & 21 \\
\hline PR0D. & 0.88 & 0.77 & 0.97 & 0.88 & 1.00 & 0.72 \\
\hline
\end{tabular}

for the following pairs of spectrally similar classes: Roads and Rooftops1, and Roads and Rooftops2. Figure 6, depicts global $\mathrm{CI}$ and the two relevant ANDI indexes. ANDI index for Grass and Trees are also shown for reference. These images show that natural classes (i.e. Trees, Grass, Water and Soil) are relatively easy to discriminate. On the contrary, Roads and Rooftops are very confusing classes.

In the Defuzzification stage, a SVM classifier with radial basis kernel was applied. Predictor variables were set to the seven fuzzy image-regions, the $\mathrm{CI}$ index and the two relevant ANDI indices (ten predictors in total), as indicated in the following formula:

$$
L A N D C O V E R=g(f 1+f 2+f 3+\ldots+f 10)
$$

where $L A N D C O V E R$ stands for the multinomial categorical response, $g$ is the SVM-based classification function, and $f 1$ to $f 10$ represent the predictor variables.

The training sample was the same set of points used for the fuzzification stage. The fitted model uses 129 support vector and a gamma value of 0.071 . Figure 7 (a) shows the final land-cover classification obtained using FIRME. As reference, a classification obtained by using a single level hard segmentation approach is shown in (b). Its apparent that FIRME results look cleaner than hard segmentation output. Global Kappa Index of Agreement (KIA) for the FIRME classification is $80 \%$. Confusion matrix is shown in Table 3. Note that confusion between Roads and Rooftops is not completely solved.

Results show that the FIRME approach leads to good accuracy for land-cover classes. Hence, it is suggested that fuzzy imageregions provide information which is potentially useful to enhance the classification of remotely-sensed images covering complex landscapes. Although the KIA index obtained in our experiments is acceptable for most practical purposes (82\%), boundaries of land-cover classes lack of a better definition and a posterior integration into a vector GIS may cause problems. A further investigation on the influence of using additional fuzzy properties and relationships on thematic accuracy should be conducted. In
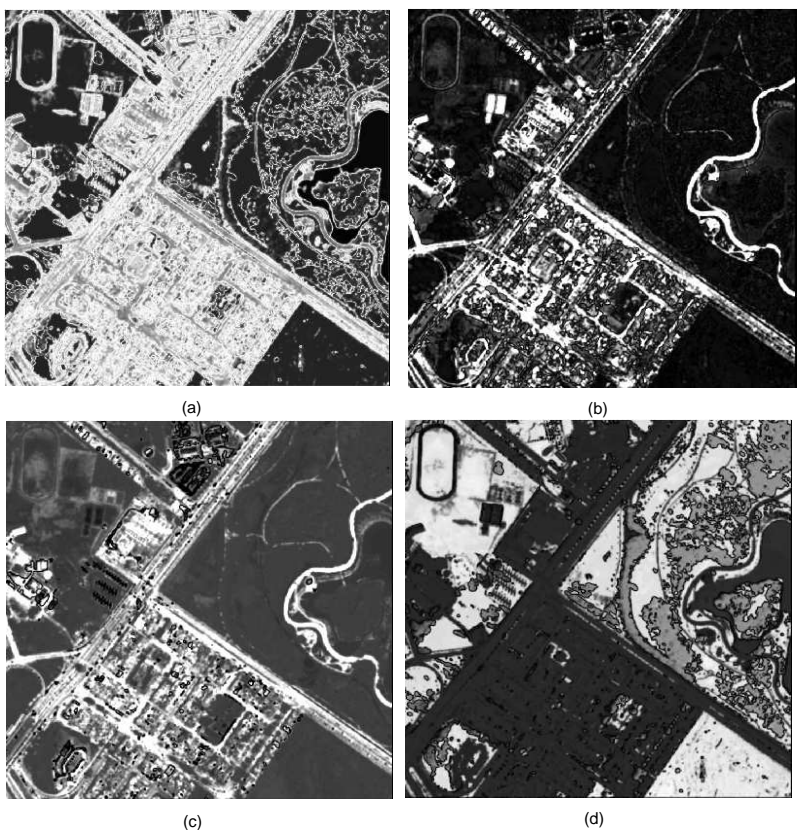

Figure 6: Images derived in the Feature Analysis stage: (a) CI index for the whole set of classes, (b) ANDI index for Roads and Rooftops1, c) ANDI index for Roads and Rooftops2, d) ANDI index for Grass and Trees. White corresponds to 1.0 and black corresponds to 0.0. Dark tones represent zones where degrees of membership are competing.

addition, post-classification methods should be also tested carefully. It has been proved that using standard techniques, like majority filtering, for eliminating noise and smoothing images, may modify susbtantially the classified image and the final accuracy (Rencai et al., 2006)

\section{CONCLUSIONS}

The contribution of this paper is summarized as follows:

- A general and flexible GEOBIA method, based on fuzzy sets, has been proposed for the classification of remotely sensed images, and

- Experimental results demonstrate that it produces good thematic accuracy in complex urban environments.

FIRME, the new method, uses Fuzzy Image-Regions properties as basis for classification. Fuzzy Image-Regions is a set of overlapping images representing degrees of memberships to competing land-cover classes. By analyzing relationships between the fuzzy image-regions contextual attributes are integrated into the vector of features used to discriminate between spectrally mixed classes. The initial fuzzification of the multispectral image and the posterior defuzzification are conducted using statistical learning methods. FIRME has been implemented using $R$ a robust statistical language and environment.

The proposed method has the following advantages compared to object-based image classification using commercial software: (i) Simplicity: Except for providing a training sample, users do not need to tweak scale or shape parameters, (ii) Flexibility: Users can choose any learning statistic method for conducting the fuzzification and/or defuzzification stages, and (iii) Low cost: Users 


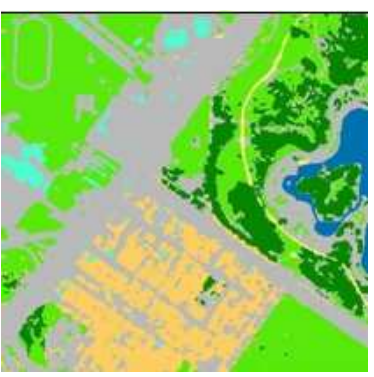

(a)

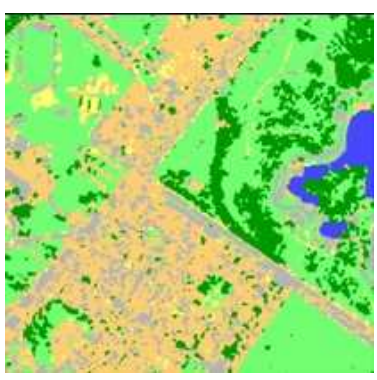

(b)
Figure 7: Final land-cover classification: (a) using FIRME method ( $\mathrm{PCC}=0.83$ ); (b) using crisp image-objects $(\mathrm{PCC}=0.70)$. Classified images show Road in gray, Roof 1 in orange, Roof 2 in cyan, Grass in light green, Trees in dark green, Water in blue and Bare Soil in yellow.

may use free open source software tools for conducting accurate remote sensing classification.

A further development of FIRME is to explore additional relationships and properties of fuzzy image-regions -only a few of them were used in the presented experiments. Hence, our future work will use geometric and contextual attributes which prove be relevant for improving the image classification process. It will also include testing the combination of SVM techniques with decision trees methods in order to produce understandable classification rules.

\section{ACKNOWLEDGEMENTS}

The multi-spectral image used in our experiments was provided by Universidad Distrital Francisco Jose de Caldas. The research reported here is partially funded by a Birkbeck International Research Studentship.

\section{REFERENCES}

Anderson, J., Hardy, E., Roach, J. and Witmer, R., 1976. A land use and land cover classification system for use with remote sensor data. Technical report, Geological Survey.

Bezdek, J., Keller, M. P. J. and Krisnauram, R., 1999. Fuzzy Models and Algorithms for Pattern Recognition and Image Processing. Springer.

Bivand, R., Pebesma, E. and Gomez-Rubio, V., 2008. Applied Spatial Data Analysis with R. Springer.

Bragato, G., 2004. Fuzzy continuous classification and spatial interpolation in conventional soil survey for soil mapping of the lower piave plain. Geoderma 118, pp. 1-16.

Burrough, P., van Gaans, P. and Hoostmans, R., 1997. Continuous classification in soil survey: spatial correlation, confusion and boundaries. Geoderma.

Chanussot, J., Nyström, I. and Sladoje, N., 2005. Shape signatures of fuzzy star-shaped sets based on distance from the centroid. Pattern Recognition Letters 26(6), pp. 735-746.

Cheng, T., Molenaar, M. and Lin, H., 2001. Formalizing fuzzy objects from uncertain classification results. International Journal of Geographical Information Science 15(1), pp. 27-42.

de Jong, S. M. and Freek, D. M., 2006. Remote Sensing Image Analysis: including the spatial domain. Springer.
Dilo, A., Bos, P., Kraipeerapun, P. and de By, R., 2006. Storage and manipulation of vague spatial objects using existing GIS functionality. In: G. Bordogna and G. Psaila (eds), Flexible databases supporting imprecision and uncertainty, Springer, pp. 293-321.

Duda, R., Hart, P. and Stork, D., 2001. Pattern Classification. Wiley.

Fisher, P. and Arnot, C., 2007. Mapping type 2 change in fuzzy land cover. In: A. Morris and S. Kokhan (eds), Geographic Uncertainty in Environmental Security, Springer, pp. 167-186.

Hastie, T., Tibshirani, R. and Friedman, J., 2001. The Elements of Statistical Learning: Data Mining, Inference and Prediction. Springer.

Jensen, J., 2006. Introductory Digital Image Processing. Prentice Hall.

Kruse, R., Doring, C. and Lesot, M., 2007. Fundamentals of fuzzy clustering. In: J. V. de Oliveira and W. Pedrycz (eds), Advances in Fuzzy Clustering and its Applications, Wiley, pp. 3-30.

Lang, S., Albretch, F. and Blaschke, T., 2006. Tutorial: Introduction to object-based image analysis. Centre for Geoinformatics Z-GIS.

Lizarazo, I. and Elsner, P., 2008. Fuzzy regions for handling uncertainty in remote sensing image segmentation. In: Computational Science and its applications ICCSA 2008 International Conference, Springer.

Lu, D. and Weng, Q., 2007. A survey of image classification methods and techniques for improving classification performance. International Journal of Remote Sensing 28(5), pp. 823870.

Mendel, J., 2001. Uncertain Rule-Based Fuzzy Logic Systems: Introduction and new directions. Prentice Hall.

Mesev, V., 2003. Remotely sensed cities. Taylor and Francis.

Rencai, D., Jiajia, D., Gand, W. and Hongbing, D., 2006. Optimization of post-classification processing of high-resolution satellite image: A case study. Science en China Series E: Technological Sciences 49(1), pp. 98-107.

Roberts, S., Hall, G. and Calami, P., 2001. Assessing polygon edge integrity. Journal of Geographical Systems pp. 87-105.

Ross, T., 2004. Fuzzy Logic with Engineering Applications. Wiley.

Schiewe, J., Tufte, L. and Ehlers, M., 2001. Potential and problems of multi-scale segmentation methods in remote sensing. GIS. Geo-Informations-Systeme 6(9), pp. 34-39.

Thomas, N., C., C. H. and Congalton, R., 2003. A comparison of urban mapping methods using high-resolution digital imagery. Photogrammetric Engineering and Remote Sensing 69(9), pp. 963-972.

Verstraete, J., A., A. H. and Tre, G. D., 2007. Fuzzy regions: Theory and applications. In: A. Morris and S. Kokhan (eds), Geographic Uncertainty in Environmental Security, Springer, pp. 117.

Wood, S., 2006. Generalized Additive Models: An Introduction to R. Chapman and Hall - CRC.

Zadeh, L., 1965. Fuzzy sets. Information and control (8), pp. 338-353.

Zinn, D., Bosch, J. and Gertz, M., 2007. Modeling and querying vague spatial objects using shapelets. In: U. of Vienna (ed.), 33rd International Conference on Very Large Databases. 